\title{
Identifying parameters in active magnetic bearing system using LFT formulation and
} Youla factorization

\author{
Lauridsen, Jonas; Sekunda, André Krabdrup; Santos, Ilmar; Niemann, Hans Henrik
}

Published in:

Proceedings of 2015 IEEE Conference on Control Applications

Link to article, DOI:

10.1109/CCA.2015.7320667

Publication date:

2015

Document Version

Peer reviewed version

Link back to DTU Orbit

Citation $(A P A)$ :

Lauridsen, J., Sekunda, A. K., Santos, I., \& Niemann, H. H. (2015). Identifying parameters in active magnetic bearing system using LFT formulation and Youla factorization. In Proceedings of 2015 IEEE Conference on Control Applications (pp. 430-435). IEEE. https://doi.org/10.1109/CCA.2015.7320667

\section{General rights}

Copyright and moral rights for the publications made accessible in the public portal are retained by the authors and/or other copyright owners and it is a condition of accessing publications that users recognise and abide by the legal requirements associated with these rights.

- Users may download and print one copy of any publication from the public portal for the purpose of private study or research.

- You may not further distribute the material or use it for any profit-making activity or commercial gain

- You may freely distribute the URL identifying the publication in the public portal 


\title{
Identifying parameters in active magnetic bearing system using LFT formulation and Youla factorization
}

\author{
Jonas S. Lauridsen ${ }^{1}$, André K. Sekunda ${ }^{2}$, Ilmar F. Santos ${ }^{3}$ and Henrik Niemann ${ }^{4}$
}

\begin{abstract}
In this paper, a method for identifying uncertain parameters in a rotordynamic system composed of a flexible rotating shaft, rigid discs and two radial active magnetic bearings is presented. Shaft and disc dynamics are mathematically described using a Finite Element (FE) model while magnetic bearing forces are represented by linear springs with negative stiffness. Bearing negative stiffness produces an unstable rotordynamic system, demanding implementation of feedback control to stabilize the rotordynamic system. Thus, to identify the system parameters, closed-loop system identification techniques are required.

The main focus of the paper relies on how to effectively identify uncertain parameters, such as stiffness and damping force coefficients of bearings and seals in rotordynamic systems. Dynamic condensation method, i.e. pseudo-modal reduction, is used to obtain a reduced order model for model-based control design and fast identification.
\end{abstract}

The paper elucidates how nodal parametric uncertainties, which are easily represented in the full FE coordinate system, can be represented in the new coordinate system of the reduced model. The uncertainty is described as a single column vector of the system matrix $A$ of the full $F E$ model while it is represented as several elements spread over multiple rows and columns of the system matrix of the reduced model. The parametric uncertainty, for both the full and reduced FE model, is represented using Linear Fractional Transformation (LFT). In this way the LFT matrices represent the mapping of the uncertainties in and out of the full and reduced FE system matrices. Scaling the LFT matrices easily leads to the amplitudes of the uncertainty parameters.

Youla Parametrization method is applied to transform the identification problem into an open-loop stable problem, which can be solved using standard optimization methods.

An example shows how to decouple and identify an uncertainty in the linear bearing stiffness of a reduced FE rotordynamic system.

\section{INTRODUCTION}

The Active Magnetic Bearing (AMB) has many advantages compared to conventional fluid film bearings and ball bearings, such as no mechanical contact, no lubrication, low maintenance, practically no friction, low vibration level and high rotational speed, which makes it extremely useful in special environments such as cleantech, subsea among

\footnotetext{
${ }^{1}$ Jonas S. Lauridsen is with Department of Mechanical Engineering, Danish University of Technology, 2800 Kgs. Lyngby, Denmark jonlau@mek.dtu.dk

${ }^{2}$ André K. Sekunda is with Department of Electrical Engineering, Control and Automation, Danish University of Technology, 2800 Kgs. Lyngby, Denmark aksek@elektro.dtu.dk

${ }^{3}$ Ilmar F. Santos is with Department of Mechanical Engineering, Danish University of Technology, 2800 Kgs. Lyngby, Denmark if samek.dtu.dk

${ }^{4}$ Henrik Niemann is with Department of Electrical Engineering, Control and Automation, Danish University of Technology, 2800 Kgs. Lyngby, Denmark hhn@elektro.dtu.dk
}

others. Today the AMB is widely used on several types of industrial applications such as centrifugal compressors, turbo expanders, blood pumps, centrifuges, machine drilling tools, energy storage flywheel etc. The AMBs have been applied in turbomachinery equipment with capacities that range from a few kilowatts up to $29,000 \mathrm{~kW}$ and with operation speed up to 60,000 RPM.

Rotors levitated by AMB's are essentially unstable systems whose properties cause several challenges to the design of active control system due to: gyroscopic effects, mass unbalance, rotor flexibility, aerodynamic excitations among others. It is essential to have a global mathematical model which precisely predicts the real plant dynamics, in order to design a high performance control system and to predict its stability and performance.

Due to assembly tolerances and simplified model assumptions, discrepancies between the model and real plant typically exist and adjustment of some of the model parameters are often needed. System identification techniques should therefore be applied to find the deviation between model and the real plant, toward more accurate global mathematical models, which in turn makes improved controller design possible.

Due to the fact that AMB systems are open-loop unstable, input-output data is only possible to gather in a closedloop scheme with a stabilizing controller. Standard open-loop identification methods are therefore ill suited [3] since they typically assume that the measurement noise is uncorrelated with the system inputs and outputs, which does not holds, once the controller action links input and output signals.

There are several methods which take into account that the plant is part of a closed loop scheme [3], [7], [14]. Each method has advantages and the method used in this paper is chosen for the easy translation to fault diagnosis of parameters. In this paper a method for closed-loop identification of the rotordynamic system (turboexpander) using a coprime factorization is proposed. The method is based on the well known Hansen scheme [17]. However classical identification using the Hansen scheme makes it difficult to take advantage of physical knowledge of the plant. The method proposed in this paper is therefore an extension which makes it possible to identify specific plant parameters through the identification of the open loop error dynamics. The method proposed in this paper has also been applied to estimate parametric faults in systems[1], [2].

The main originality of the work relies on parametrization and identification of uncertainties in FE rotordynamic systems. Focus is put on how LFT representation of a reduced 
system can be obtained based on a full FE representation of a rotordynamic system.

This paper deals with a $700 \mathrm{~kW}$ turboexpander supported by AMB designed for air separation units. The turboexpander can essentially be considered as a flexible rotor spinning at angular velocities up to 31500 RPM, levitated by AMB forces. In this paper the modelling of the shaft is carried out using FE method including gyroscopic effects [11], [12], and the forces of the AMB have been characterized using the basic laws of electromagnetism [10].

The paper is structured as follows: Section II introduces the identification method of unstable systems based on Youla parmetrization; Section III contains a description of the system to be identified, modelling and reduction of the system, followed by representation of the uncertainty; Section IV discuss results obtained from a simulation example of applying the identification method to a plant with parameter uncertainties; Section V contains a conclusion on the results presented in the paper.

\section{METHOD}

\section{Identification of system using Coprime factorization}

In this section a method for identification of closed-loop systems using coprime factorization is given. The method uses the coprime factorisation of plant and controller and is based on the theory outlined in [4]. The closed-loop scheme is given as shown in Fig. 1 . Let $G=G(0)$ be the nominal

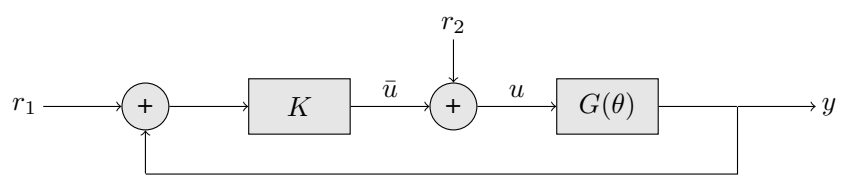

Fig. 1. Closed-loop system representation.

plant i.e. initial model guess and $K$ be a stabilising controller to both the real plant $G(\theta)$ and the nominal plant $G$, where $\theta$ is the parameter uncertainty. Then $G$ and $K$ are given as:

$$
\begin{aligned}
& G=N M^{-1}=\tilde{M}^{-1} \tilde{N} \\
& K=U V^{-1}=\tilde{V}^{-1} \tilde{U}
\end{aligned}
$$

For the 8 matrices given in Eq. (1) and Eq. (2) to be coprime factors, the double Bezout identity shown in Eq. (3) have to be satisfied.

$$
\left[\begin{array}{ll}
I & 0 \\
0 & I
\end{array}\right]=\left[\begin{array}{cc}
\tilde{V} & -\tilde{U} \\
-\tilde{N} & \tilde{M}
\end{array}\right]\left[\begin{array}{ll}
M & U \\
N & V
\end{array}\right]=\left[\begin{array}{cc}
M & U \\
N & V
\end{array}\right]\left[\begin{array}{cc}
\tilde{V} & -\tilde{U} \\
-\tilde{N} & \tilde{M}
\end{array}\right]
$$

With a coprime factorization of the nominal plant $G$ and of the controller $K$ stabilizing both the real plant $G(\theta)$ and the nominal plant $G(0)$, Eq. (4) gives a parametrization of all stabilizing controllers, for the nominal plant, using the stable transfer matrix $Q$, from $\epsilon$ to $\eta$ shown in Fig. 2 [4].

$$
K(Q)=(\tilde{V}+Q \tilde{N})^{-1}(\tilde{U}+Q \tilde{M})
$$

This controller can be represented as a LFT [6].

$$
\begin{aligned}
K(Q) & =\mathcal{F}_{l}\left(\left[\begin{array}{cc}
U V^{-1} & \tilde{V}^{-1} \\
V^{-1} & -V^{-1} N
\end{array}\right], Q\right) \\
& =\mathcal{F}_{l}\left(J_{k}, Q\right)
\end{aligned}
$$

Equivalent, all plants stabilized by $\mathrm{K}$ can be parameterized

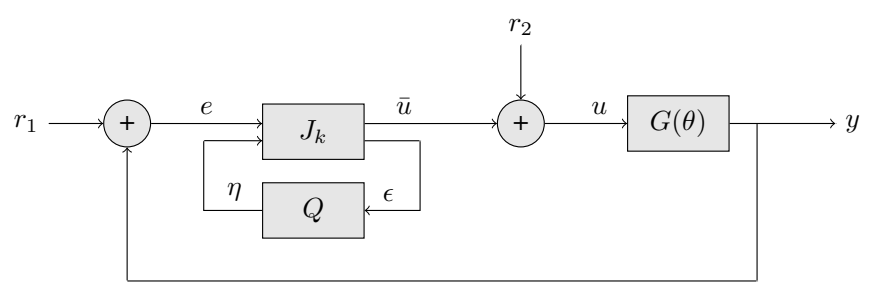

Fig. 2. Closed-loop system representation with all stabilizing controllers parametrised using a stable transfer matrix $Q$.

as Eq. (9). Taking advantage of the relationship given in Eq. (7) between the parametrized controller $K(Q)$ and the parametrized plant $G(S)$ [4], it is possible to show that Eq. (9) is a parametrization of all plants stabilized by the controller $K$ using the stable system $S(\theta)$ being the transfer matrix from $\eta$ to $\epsilon$ shown in Fig. 2.

$$
\begin{aligned}
S & =\mathcal{F}_{l}\left(J_{k}, G(S)\right) \\
\epsilon & =S(\theta) \eta \\
G(S) & =(\tilde{M}+S \tilde{U})^{-1}(\tilde{N}+S \tilde{V})^{-1}
\end{aligned}
$$

If the nominal plant is equal to the real plant, $S(\theta)$ is zero. As the nominal plant differs from the real plant, $S(\theta)$ increases and can thus be considered as a expression of the deviation between the nominal and the real plant.

A standard Luenberger observer is used for implementation of $S(\theta)$ for simulation examples. However any controller with its associated coprime factorization can be used. For a system such as shown in Fig. 2, the closed-loop transfer function can be written as [1].

$$
\begin{gathered}
{\left[\begin{array}{l}
y \\
u \\
\epsilon
\end{array}\right]=T_{c l}(S)\left[\begin{array}{c}
r_{1} \\
r_{2} \\
\eta
\end{array}\right]} \\
T_{c l}(S)=\left[\begin{array}{ccc}
(N+V S) \tilde{U} & (N+V S) \tilde{V} & N+V S \\
(M+U S) \tilde{U} & (M+U S) \tilde{V} & M+U S \\
\tilde{M}+S \tilde{V} & \tilde{N}+S \tilde{U} & S
\end{array}\right]
\end{gathered}
$$

With input and output of the system defined, the uncertainties need to be given in regards to $S(\theta)$. Parameter uncertainties are given using a LFT description. Plant uncertainties are therefore given as in Eq. (12).

$$
G(\theta)=\mathcal{F}_{l}\left(\left[\begin{array}{ll}
G_{y u} & G_{y w} \\
G_{z u} & G_{z w}
\end{array}\right], \theta\right)
$$

Here $\theta$ is a diagonal matrix with a parameter uncertainty in each diagonal element. It is worth noticing that $G(0)$ is equivalent to the nominal plant. A description of how to represent the parameter uncertainties as an LFT is shown in 


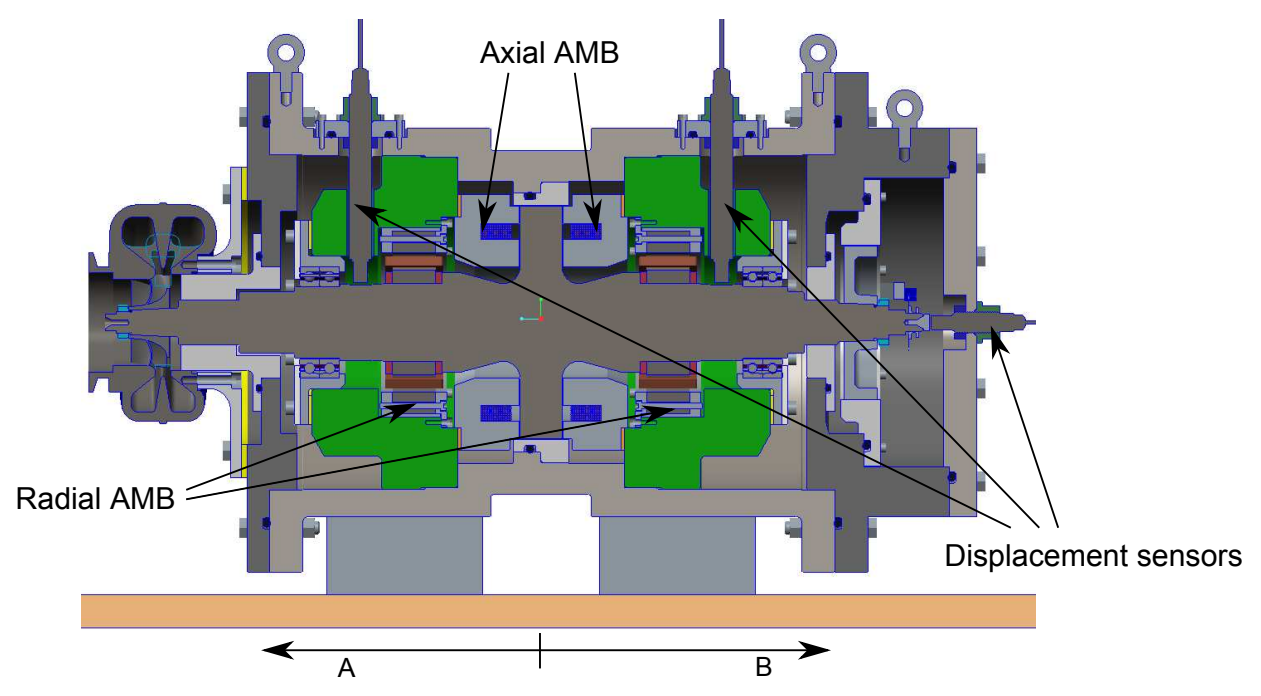

Fig. 3. Cross-section of the turboexpander testrig.

Section III-.7. With the uncertainties defined as in Eq. (12), $S(\theta)$ is found in [1] to be

$$
S(\theta)=\mathcal{F}_{l}\left(\left[\begin{array}{cc}
0 & \tilde{M} G_{y w} \\
G_{z u} M & G_{z w}+G_{z u} U \tilde{M} G_{y w}
\end{array}\right], \theta\right)
$$

Due to $\eta$ not being correlated with the disturbances $r_{1}$ and $r_{2}$, Eq. (10) can be used for identification of the open loop error $S$. Estimation of the open loop error $S$ from $\eta$ to $\epsilon$ simplifies to Eq. (14), which can be considered as an openloop identification problem of the stable system $S(\theta)$ with uncorrolated noise in the prediction [4].

$$
\epsilon=S(\theta) \eta+D_{1} r_{1}+D_{2} r_{2}
$$

Identification of parameter uncertainties using a LFT scheme is a well studied subject in open-loop identification of Linear Parameter Varying (LPV) systems [7], [8], [9]. The approach is to define a cost function and minimize the error between the measured and calculated output of the system. The cost function is given in Eq. (15) in its approximate quadratic form.

$$
J(\theta)=\int_{0}^{t} \frac{1}{2}(\epsilon-S(\theta) \eta)^{2} d t
$$

The goal is to find the global minima of (15) which can be done using several different methods. In this paper the Matlab function fminsearch is used, which is an general unconstrained nonlinear optimization method. Other methods, like gradient methods, has shown to yield faster convergence for specific types of plants, however this has not been the main focus.

\section{SYSTEM AND UNCERTAINTY REPRESENTATION}

In this section, the rotordynamic system is described and it is shown how dynamic uncertainties from such a system can be extracted and represented in Linear Fractional Transformation (LFT) form.
1) The real system: A cross-section schematic of the turboexpander investigated is shown in Fig. 3. The turboexpander essentially consists of a shaft levitated using axial and radial AMBs. It is assumed that the only forces acting on the rotor are the left and right radial AMB. The displacement sensors are placed close to the AMBs. The placement of the sensors and actuators will be denoted by $A_{x}, A_{y}$ and $B_{x}$, $B_{y}$.

The analysis will be focused on rotor lateral movements and for simplicity the rotor axial movements will not be investigated. The term AMB will therefore refer to the radial AMBs in the following.

2) Model of AMBs: The model of the magnetic bearing is simplified to describe the forces acting on the rotor as function of the rotor lateral displacements $s$ and the control current $i_{x}$. The linearized expression of the forces are given as [10]

$$
f_{b}\left(i_{x}, s\right)=K_{i} i_{x}+K_{s} s
$$

where $K_{i}$ are $K_{s}$ are constants. $K_{s}$ can be considered as the stiffness of the bearing forces which is negative and thus makes the system open-loop unstable. The dynamics of the electromechanical system including the inductance of the coil and the amplifiers have been neglected.

3) Model of shaft: The rotating shaft has been modelled using the FE method and Bernoulli-Euler beam theory taking into account the gyroscopic effects of the shaft and discs

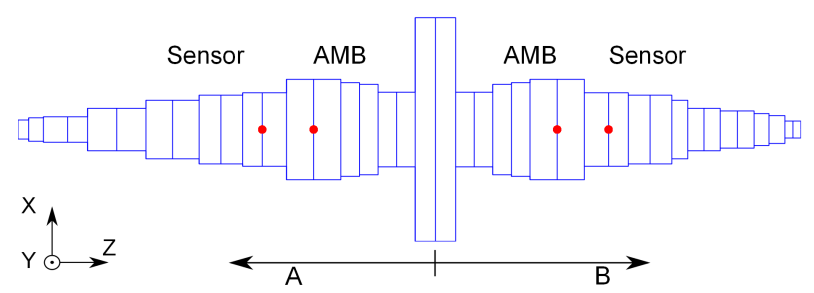

Fig. 4. Discretization of the shaft. Placement of sensors and AMBs are shown. 
[11], [12]. The shaft have been dicretized in 40 node points with 4 degrees of freedom each, which is $\mathrm{x}$ and $\mathrm{y}$ direction, and the rotation around the $\mathrm{x}$ and $\mathrm{y}$ axes, which yields 320 states in total. The discretization of node points of the shaft and the placement of sensors and AMBs is shown in Fig. 4. The goal is to control the rigid body motion of the rotating shaft and it is possible to obtain a reduced model of the rotor-bearing system with 8 states by using pseudomodal reduction [15], [16] and removing all flexible modes, described in the following section. The reduction method are later used for LFT representation of uncertainties in the reduced FE plant model G.

Hence a MIMO system with 4 inputs (control current) and 4 outputs (rotor displacement) and 8 states have been obtained.

4) Model Reduction: The full order rotordynamic system $G_{f}$ consisting of the finite element model of the shaft and negativ stiffness forces from the AMB can be written in state space form

$$
\dot{x}_{f}=A_{f} x_{f}+B_{f} u, \quad y=C_{f} x_{f}
$$

The system left and right eigenvectors $\left(U_{l}\right.$ and $\left.U_{r}\right)$ are found by solving the eigenvalue problem

$$
\begin{aligned}
& A_{f} U_{r}=\lambda U_{r} \\
& A_{f}^{T} U_{l}=\lambda U_{l}
\end{aligned}
$$

The system can be sorted by the undamped natural frequencies, $|\mathcal{I}(\lambda)|$, since only the low frequency dynamics are of interest. The eigenvectors for the corresponding eigenvalues are used to create right and left transformation matrices

$$
\begin{aligned}
T_{r} & =\left[\begin{array}{llll}
U_{r_{1}} & U_{r_{2}} & \ldots & U_{r_{n}}
\end{array}\right] \\
T_{l} & =\left[\begin{array}{llll}
U_{l_{1}} & U_{l_{2}} & \ldots & U_{l_{n}}
\end{array}\right]
\end{aligned}
$$

The reduced system is then given as

$$
\dot{x}_{c}=A_{c} x_{c}+B_{c} u_{c}, \quad y_{c}=C_{c} x_{c}
$$

where

$$
\begin{aligned}
& x_{c}=T_{l}^{T} x_{f} \\
& A_{c}=T_{l}^{T} A_{f} T_{r} \\
& B_{c}=T_{l}^{T} B_{f} \\
& C_{c}=C_{f} T_{r}
\end{aligned}
$$

In this way the system is decomposed into a reduced system $A_{c}$ which contains the dominant dynamics and the residual system $A_{\text {res }}$ containing the residual dynamics, as shown below

$$
\left[\begin{array}{c}
\dot{x}_{c} \\
\dot{x}_{r e s}
\end{array}\right]=\left[\begin{array}{cc}
A_{c} & 0 \\
0 & A_{\text {res }}
\end{array}\right]\left[\begin{array}{c}
x_{c} \\
x_{r e s}
\end{array}\right]+\left[\begin{array}{c}
B_{c} \\
B_{r e s}
\end{array}\right] u
$$

Fig. 5 shows the singular values of the full and the reduced system. It is seen that the reduced 8 states system fits the dynamics very well up to approx. $20 \times 10^{3} \mathrm{rad} / \mathrm{s}$ which is above the frequency range of interest. The singular values are shown for the rotordynamic system when angular velocity is 31500 RPM since the system identification is assumed to take place at nominal operational conditions.

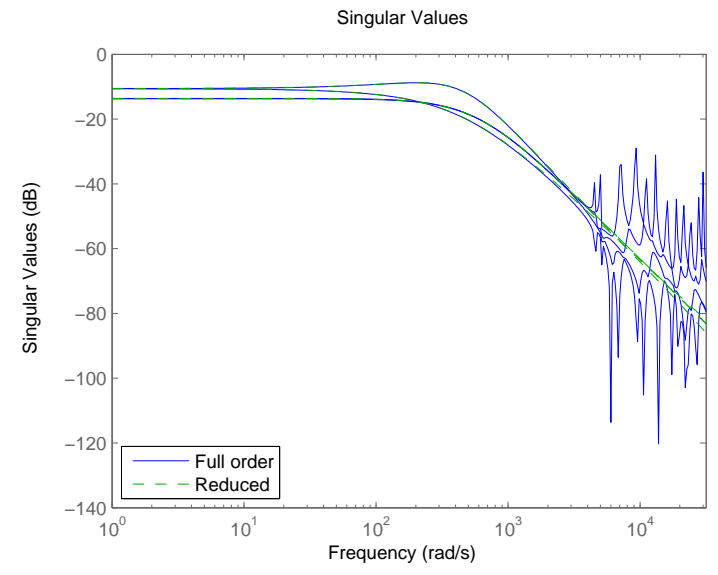

Fig. 5. Singular values of the full and the reduced rotor dynamic system shown at nominal angular velocity of 31500 RPM

5) Complex separation: The reduced state space model obtained by modal reduction consist of complex coefficients. This model can be rewritten to real form with $2 n$ states, one state to represent the real part and one for the imaginary part [13]. This can be done by transforming the $T_{l}^{T}$ and $T_{r}$ to

$$
\begin{aligned}
& T_{r_{\text {sep }}}=\left[\begin{array}{lllll}
\mathcal{R}\left(T_{r_{1}}\right) & -\mathcal{I}\left(T_{r_{1}}\right) & \ldots & \mathcal{R}\left(T_{r_{n}}\right) & -\mathcal{I}\left(T_{r_{n}}\right)
\end{array}\right] \\
& T_{l_{\text {sep }}}=\left[\begin{array}{lllll}
\mathcal{R}\left(T_{l_{1}}\right) & \mathcal{I}\left(T_{l_{1}}\right) & \ldots & \mathcal{R}\left(T_{l_{n}}\right) & \mathcal{I}\left(T_{l_{n}}\right)
\end{array}\right]
\end{aligned}
$$

Such that the new system $G_{s}$ with new state vector $x_{s}$ and the matrices $A_{s}, B_{s}$ and $C_{s}$ are given as

$$
\begin{aligned}
& \dot{x}_{s}=A_{s} x_{s}+B_{s} u, \quad y=C_{s} x_{s} \\
& x_{s}=\left[\begin{array}{lllll}
\mathcal{R}\left(x_{c_{1}}\right) & \mathcal{I}\left(x_{c_{1}}\right) & \ldots & \mathcal{R}\left(x_{c_{n}}\right) & \mathcal{I}\left(x_{c_{n}}\right)
\end{array}\right]^{T}, \\
& A_{s}=\left[\begin{array}{lll}
\ddots & & 0 \\
& A_{c_{i i}} & \\
0 & & \ddots
\end{array}\right], \quad A_{c_{i i}}=\left[\begin{array}{cc}
\mathcal{R}\left(\lambda_{i}\right) & -\mathcal{I}\left(\lambda_{i}\right) \\
\mathcal{I}\left(\lambda_{i}\right) & \mathcal{R}\left(\lambda_{i}\right)
\end{array}\right] \text {, } \\
& B_{s}=\left[\mathcal{R}\left(B_{c_{1}}\right), \mathcal{I}\left(B_{c_{1}}\right) \ldots \mathcal{R}\left(B_{c_{n}}\right), \mathcal{I}\left(B_{c_{n}}\right)\right]^{T}, \\
& C_{s}=\left[\mathcal{R}\left(C_{c_{1}}\right),-\mathcal{I}\left(C_{c_{1}}\right) \ldots \mathcal{R}\left(C_{c_{n}}\right),-\mathcal{I}\left(C_{c_{n}}\right)\right],
\end{aligned}
$$

6) Reduction of uncontrollable and unobservable modes: After complex separation the system consist of $2 n$ states. By considering which states that is controllable and which are observable it becomes clear that some states are uncontrollable and can be removed. A similarity transform $T_{\text {sim }}$ exists which transforms the complex separated system $G_{s}$ into a controllable part and an uncontrollable part which can be removed

$$
\begin{aligned}
& \bar{A}=T_{\text {sim }} A_{s} T_{\text {sim }}^{T} \\
& \bar{B}=T_{\text {sim }} B_{s} \\
& \bar{C}=C_{s} T_{\text {sim }}^{T}
\end{aligned}
$$

and the transformed system has the form

$$
\bar{A}=\left[\begin{array}{cc}
A_{n c o n} & 0 \\
A_{21} & A_{\text {con }}
\end{array}\right], \bar{B}=\left[\begin{array}{c}
0 \\
B_{c o n}
\end{array}\right], \bar{C}=\left[\begin{array}{c}
0 \\
B_{c o n}
\end{array}\right]
$$


where $A_{\text {con }}, B_{c o n}, C_{c o n}$ represents the controllable system.

The final transformation matrices denoted $T_{R}$ and $T_{L}$ can thus be found as the lower part of the products $T_{r_{\text {sep }}} T_{\text {sim }}^{T}$ and $T_{l_{\text {sep }}}^{T} T_{\text {sim }}$

$$
\begin{aligned}
T_{r_{*}} & =T_{r_{s e p}} T_{s i m}^{T} \\
T_{l_{*}} & =T_{\text {sim }} T_{l_{\text {sep }}}^{T} \\
T_{R} & =T_{r_{*}}(:, n+1: 2 n) \\
T_{L} & =T_{l_{*}}(n+1: 2 n,:)
\end{aligned}
$$

Thus the final reduced system matrices can be written as

$$
\begin{aligned}
& A=T_{L} A_{f} T_{R} \\
& B=T_{L} B_{f} \\
& C=C_{f} T_{R}
\end{aligned}
$$

The transformation matrices $T_{R}$ and $T_{L}$ will later be used to map the uncertainty from the full system to the reduced system.

7) Identification of parameter uncertainty using LFT of full system: LFT can be used for representing a nominal system with a parameter uncertainty. A lower LFT can be written as [6]

$$
\mathcal{F}_{l}(G, \theta)=G_{y u}+G_{y w} \theta\left(I-G_{z w} \theta\right)^{-1} G_{z u}
$$

If $G_{z w}$ is zero, the LFT representation can be simplified to

$$
\mathcal{F}_{l}(G, \theta)=G_{y u}+G_{y w} \theta G_{z u}
$$

$G_{y w}$ and $G_{z u}$ can be considered as the mapping of the uncertainty in and out of the the states of the system, where $G_{y u}$ can be considered as the nominal system as if the uncertainty is zero $(G(0))$.

It is chosen to investigate the possibility of identifying the uncertainty of a parameter in the system. A change in the negative bearing stiffness in a single direction, in a single position, is considered, which happens at e.g. $B_{y}$, see Fig. 4.

It is therefore investigated if the change in negative stiffness can be described by an LFT using $G_{y w_{f}}$ and $G_{z u_{f}}$ scaled by $\theta$, on the form shown in Eq. (47). The subscript $f$ denotes the full system i.e. the full finite element system with 40 nodes and 320 states (before model reduction). It can be proved that a change in stiffness (or damping) at a single direction at e.g. $B_{y}$ corresponds to a change in a single column of system matrix A, which corresponds to the node $j$ where the stiffness has changed.

$$
A_{\Delta_{f}}=\left[\begin{array}{ccccccc}
0 & \ldots & 0 & a_{1, j} & 0 & \ldots & 0 \\
0 & \ldots & 0 & a_{2, j} & 0 & \ldots & 0 \\
\vdots & \ddots & \vdots & \vdots & \vdots & \ddots & \vdots \\
0 & \ldots & 0 & a_{i, j} & 0 & \ldots & 0
\end{array}\right]
$$

$G_{y w_{f}}$ and $G_{z u_{f}}$ can then easily be obtained by selecting $G_{y w_{f}}$ to be the column of system matrix A which has changed

$$
G_{y w_{f}}=\left[\begin{array}{c}
a_{1, j} \\
a_{2, j} \\
\vdots \\
a_{i, j}
\end{array}\right]
$$

and select $G_{z u}$ to be

$$
G_{z u_{f}}=\left[\begin{array}{lllllll}
0 & 0 & \ldots & 1 & \ldots & 0 & 0
\end{array}\right]
$$

where 1 should be placed at the position of column which has changed in A (node position). $\theta$ is simply selected to 1 which would correspond to a $100 \%$ change in the system parameter.

8) LFT of reduced system: The LFT of the reduced system can now simply be described using the transformation matrices given in Eq. (41) and Eq. (42) to transform the uncertainty mapping $G_{y w_{f}}$ and $G_{z u_{f}}$ from the full finite element system to the reduced system on modal form given by Eq. (43).

$$
\begin{gathered}
G_{y w}=T_{L} G_{y w_{f}} \\
G_{z u}=G_{z u_{f}} T_{R} \\
\text { IV. RESULTS }
\end{gathered}
$$

\section{RESULTS}

This section demonstrates that it is possible to identify an uncertainty using the method introduced in Section II on a rotordynamic system and uncertainty representation as presented in Section III.

Before identification of the plant is conducted, it is shown why open-loop identification of the plant is not possible. On Fig. 6 the poles and zeros of real plant $G(\theta)$ is shown, hence the real plant to be identified. It is easy to see that any input given to the plant would make the output increase to infinity, due to poles in the right half plane. Such right half plane poles are not present in $S(\theta)$, as can be seen in Fig. 7, why open-loop identification of $S(\theta)$ is possible.

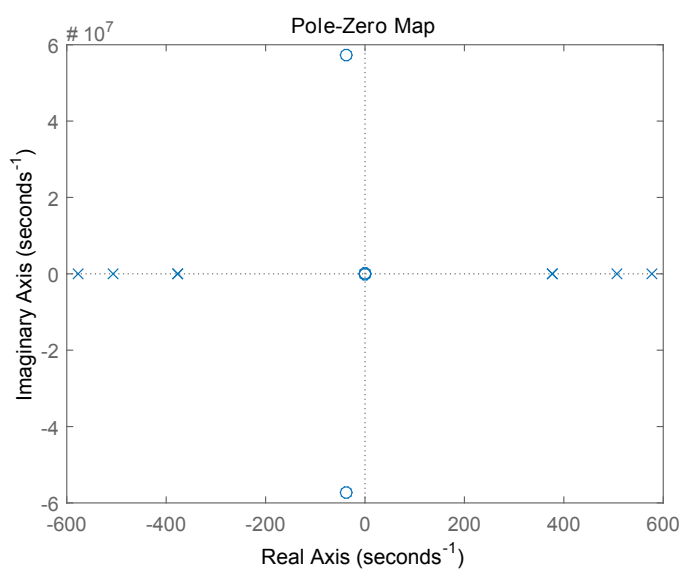

Fig. 6. Pole-Zero plot of $G(\theta)$. Poles are marked using $\times$ 's and zeros are marked using o's.

A simulation is conducted with a controller stabilizing both nominal model plant and the real plant. A stiffness reduction 


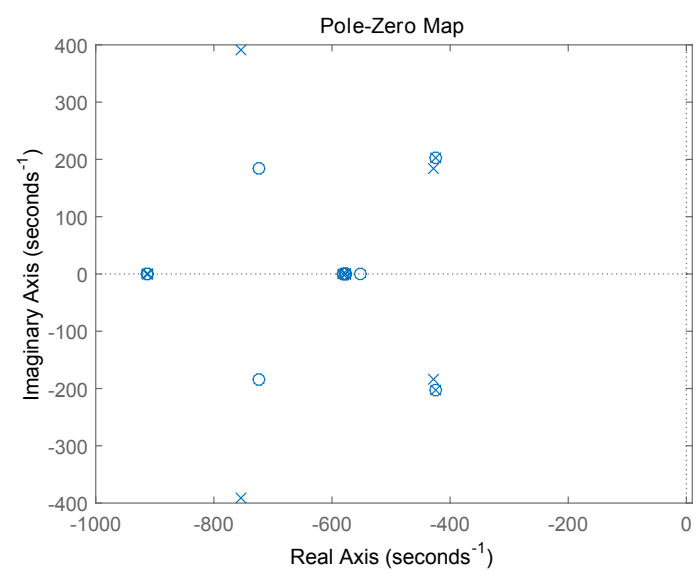

Fig. 7. Pole-Zero plot of $S(\theta)$. Poles are marked using $\times$ 's and zeros are marked using o's.

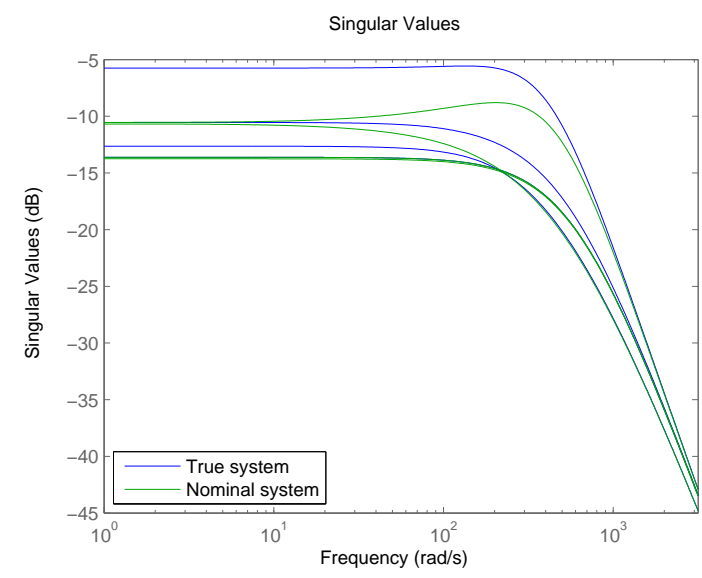

Fig. 8. Comparison of the singular values of the real system to be identified and the nominal system

of $50 \%(\theta=0.5)$ is introduced to the real plant compared to the nominal plant model.

The frequency response of the nominal and real plants are shown in Fig. 8. The plot shows that the uncertainty injected through the LFT change the dynamics of the system.

For the identification, a random binary signal is chosen for $\eta$ and both $r_{1}$ and $r_{2}$ are set to 0 . The variables $\eta, r_{1}$ and $r_{2}$ are shown in Fig. 2. A time period of $0.5 \mathrm{~s}$ and a time step of $0.001 \mathrm{~s}$ are chosen. The uncertainty, $\theta$, is identified to be 0.502 which is practically the same as the theoretical result $(\theta=0.5)$.

\section{CONCLUSION}

The problem of estimating uncertain dynamics in a rotordynamic system supported by AMB is considered. Finite element and modal reduction methods are applied to establish a reduced model of the system and to parametrize uncertain dynamics in the system into uncertain parameters, which then can be identified. Youla parametrization theory is applied to show how the unstable system in connection with a standard observer based feedback structure can be used to transform the identification problem into an open loop stable formulation describing the change of dynamics between the modelled system and the real system. This method is proposed for rotordynamic systems, in which the finite element model of shaft is known in advance, but where e.g. bearing or seal dynamics is uncertain.

From the example it can be concluded that the method works when considering an ideal case where the bearing stiffness in one direction is uncertain. The ideal case is used to give a clear overview of the methodology proposed. The example shows that the bearing stiffness is efficiently identified, while the shaft is spinning at nominal angular speed.

There are various possibilities to be investigated with this method such as to extend the shaft model to include flexible modes, identify multiples parameters simultaneously, investigate the effect of disturbances, investigate the effect of uncertain shaft dynamics and carry out experimental tests.

\section{REFERENCES}

[1] Niemann H. and Poulsen N. K, Estimation of parametric fault in closed-loop systems, 2015 American Control Conference(ACC) June 1-3, Chicago, Illinois, USA.

[2] Niemann H., Stoustrup J., and Poulsen N. K, "Controller modification applied for active fault detection." American Control Conference (ACC), 2014.

[3] Anderson B. D. O., "From Youla Kucera to identification, adaptive and nonlinear control." Automatica 34.12 1998: 1485-1506.

[4] Tay, T. T., Mareels I., and Moore J. B., "High performance control" Springer Science \& Business Media, 1998.

[5] Niemann H., "Architecture for fault diagnosis and fault-tolerant control", Department of Electrical Engineering DTU, 2015.

[6] Zhou K., and Doyle J. C. "Essentials of robust control", Vol. 180. Upper Saddle River, NJ: Prentice hall, 1998.

[7] Wolodkin G., Rangan S. and Poolla K. "An LFT approach to parameter estimation." American Control Conference, 1997. Proceedings of the 1997. Vol. 3. IEEE, 1997.

[8] Hsu K, et al. "An LFT approach to parameter estimation." Automatica 44.12 (2008): 3087-3092.

[9] Casella F. and Marco L., "LPV/LFT modelling and identification: overview, synergies and a case study." Computer-Aided Control Systems, 2008. CACSD 2008. IEEE International Conference on. IEEE, 2008.

[10] H Bleuler, et al. "Magnetic bearings: theory, design, and application to rotating machinery", Eds. Gerhard Schweitzer, and Maslen E. H., Springer Science \& Business Media, 2009.

[11] Nelson, H. D., and McVaugh, J. M., "The dynamics of rotor-bearing systems using finite elements." Journal of Manufacturing Science and Engineering 98.2 (1976): 593-600.

[12] Nelson, H. D., "A finite rotating shaft element using Timoshenko beam theory." Journal of mechanical design 102.4 (1980): 793-803.

[13] Christensen, R. H., and Santos I. F., "Design of active controlled rotorblade systems based on time-variant modal analysis." Journal of sound and vibration 280.3 (2005): 863-882.

[14] Söderström T., and Stoica P. "System Identification", London, UK: Prentice hall, 1989.

[15] Roehrle, H. (1980) "Reduktion von Freiheitsgraden bei Strukturdynamikaufgaben", VDI Reihe 1, Nr. 72.

[16] Bucher, C. (1985) "Contributions to the Modeling of Flexible Structures for Vibration Control", PhD Thesis, Swiss Federal Institute of Technology Zrich, Switzerland.

[17] Hansen F., Franklin G. and Kosut Robert(1989), Closed-Loop Identification via the Fractional Representation: Experiment Design, In Proc. Amer. Control Conf., pp. 386-391 